\title{
Wireless control of intraspinal microstimulation in a rodent model of paralysis
}

\author{
*Peter J. Grahn, BA, ${ }^{1}$ Kendall H. Lee, MD, PhD, ${ }^{2,3}$ Aimen Kasasbeh, MD, PhD, ${ }^{2}$ \\ Grant W. Mallory, MD, ${ }^{2}$ Jan T. Hachmann, ${ }^{1,2}$ John R. Dube, BS, ${ }^{2}$ Christopher J. Kimble, MA, ${ }^{4}$ \\ Darlene A. Lobel, MD, ${ }^{2}$ Allan Bieber, PhD, ${ }^{1,2,5}$ Ju Ho Jeong, MD, PhD, ${ }^{2}$ \\ Kevin E. Bennet, BSChE, MBA, ${ }^{2,4}$ and J. Luis Lujan, $\mathrm{PhD}^{2,3}$
}

${ }^{1}$ Mayo Graduate School, Departments of ${ }^{2}$ Neurologic Surgery and ${ }^{3}$ Physiology and Biomedical Engineering, ${ }^{4}$ Division of Engineering, and ${ }^{5}$ Department of Neurology, Mayo Clinic, Rochester, Minnesota

OBJECT Despite a promising outlook, existing intraspinal microstimulation (ISMS) techniques for restoring functional motor control after spinal cord injury are not yet suitable for use outside a controlled laboratory environment. Thus, successful application of ISMS therapy in humans will require the use of versatile chronic neurostimulation systems. The objective of this study was to establish proof of principle for wireless control of ISMS to evoke controlled motor function in a rodent model of complete spinal cord injury.

METHODS The lumbar spinal cord in each of 17 fully anesthetized Sprague-Dawley rats was stimulated via ISMS electrodes to evoke hindlimb function. Nine subjects underwent complete surgical transection of the spinal cord at the T-4 level 7 days before stimulation. Targeting for both groups (spinalized and control) was performed under visual inspection via dorsal spinal cord landmarks such as the dorsal root entry zone and the dorsal median fissure. Teflon-insulated stimulating platinum-iridium microwire electrodes (50 $\mathrm{um}$ in diameter, with a 30 - to 60-um exposed tip) were implanted within the ventral gray matter to an approximate depth of $1.8 \mathrm{~mm}$. Electrode implantation was performed using a freehand delivery technique $(n=12)$ or a Kopf spinal frame system $(n=5)$ to compare the efficacy of these 2 commonly used targeting techniques. Stimulation was controlled remotely using a wireless neurostimulation control system. Hindlimb movements evoked by stimulation were tracked via kinematic markers placed on the hips, knees, ankles, and paws. Postmortem fixation and staining of the spinal cord tissue were conducted to determine the final positions of the stimulating electrodes within the spinal cord tissue.

RESULTS The results show that wireless ISMS was capable of evoking controlled and sustained activation of ankle, knee, and hip muscles in $90 \%$ of the spinalized rats $(n=9)$ and $100 \%$ of the healthy control rats $(n=8)$. No functional differences between movements evoked by either of the 2 targeting techniques were revealed. However, frame-based targeting required fewer electrode penetrations to evoke target movements.

CONCLUSIONS Clinical restoration of functional movement via ISMS remains a distant goal. However, the technology presented herein represents the first step toward restoring functional independence for individuals with chronic spinal cord injury.

http://thejns.org/doi/abs/10.3171/2014.10.JNS132370

KEY WORDS intraspinal microstimulation; functional electrical stimulation; spinal cord injury; diagnostic and operative techniques

$\mathrm{T}$ Raumatic spinal cord injury (SCI) is a devastating condition that can result in permanent loss of sensorimotor and autonomic function and can significantly reduce the quality of life for affected individuals.
Spinal injury carries a large economic impact extending over the lifespan of a patient, with an estimated cost of up to $\$ 1,000,000$ for the 1 st year after injury alone (see https:// www.nscisc.uab.edu). Approximately 300,000 people are

ABBREVIATIONS EMG = electromyographic; ISMS = intraspinal microstimulation; MINCS = Mayo investigational neuromodulation control system; SCI = spinal cord injury. SUBMITTED October 25, 2013. ACCEPTED October 14, 2014.

INCLUDE WHEN CITING Published online December 5, 2014; DOI: 10.3171/2014.10.JNS132370.

DISCLOSURE Dr. Lujan is a patent holder with Boston Scientific. The following sources funded this study: Grant Nos. R01 NS 75013 and R01 NS 70872 from the National Institutes of Health (to K.H.L.), Grant No. R21 NS087320 (to J.L.L.), a grant from The Grainger Foundation (to K.H.L.), and a gift from Louise Chapman (to K.H.L.).

${ }^{*}$ Mr. Grahn and Dr. Lee contributed equally to this work. 
currently living with SCI, and 12,000 new cases are reported annually in the United States. ${ }^{3}$ In healthy individuals, the intact CNS initiates and coordinates movement through electrical signals traveling from the brain to the muscles via corticospinal pathways. However, SCI prevents transmission of these signals to effector motor neurons in the spinal cord, resulting in muscle paralysis below the level of injury.

Spinal motor networks below the injury level remain intact and are capable of driving motor function when electrically stimulated. ${ }^{14}$ As such, electrical stimulation of peripheral nerves and skeletal muscles has been widely used to restore function to paralyzed muscles. . $^{4,10,16,17,31}$ However, the functional benefits of these techniques are overshadowed by significant practical limitations, including reverse motor unit recruitment, ${ }^{6}$ early fatigue onset, ${ }^{24}$ low muscle force, stimulation spillover, and difficulty associated with donning or doffing of stimulation devices. ${ }^{25,29}$ More recently, stimulation of the spinal cord via intraspinal microelectrodes has been used successfully to evoke limb movement in rodent and feline models of acute SCI. ${ }^{1,15,19,21,23,26}$ Intraspinal microstimulation (ISMS) is believed to work by directly stimulating effector motor circuitry in the ventral spinal cord, thus achieving a more natural recruitment of motor units. ${ }^{13}$ In turn, this recruitment offers better resistance to muscle fatigue than peripheral nerve and muscle stimulation techniques. ${ }^{1,22,23,27}$ In humans, epidural spinal stimulation was recently used to achieve full weight-bearing standing in a 23-year-old man suffering from complete paraplegia. ${ }^{12}$ Unfortunately, epidural stimulation lacks the selectivity required to evoke complex movements. Similarly, state-of-the-art intraspinal stimulation techniques are hindered by the use of conventional hard-wired stimulators that limit the conditions under which this technology can be tested. Thus, it is clear that effective clinical translation of restorative technologies such as ISMS requires not only high-resolution coordinated muscle activation but also improved fatigue resistance and the ability to evaluate novel stimulation paradigms in unrestricted settings. In this proof-of-principle study, we address fatigue resistance by repeatedly administering wireless control of ISMS in intact and spinal cord-injured rodents. In addition, we describe some important limitations of the existing ISMS technology that prevent the clinical restoration of complex motor function. The wireless ISMS system described herein represents a platform for developing and testing novel stimulation paradigms and technologies for simultaneous stimulation and behavioral analysis of evoked responses. As such, wireless neurostimulation represents the first step toward achieving the clinical restoration of complex motor function by activating paretic muscles outside conventional laboratory constraints.

\section{Methods \\ Rats}

Studies were conducted in vivo in 17 adult female Sprague-Dawley rats weighing 225-275 g. Of these animals, 9 were used as an SCI model and 3 were used as intact healthy controls. The remaining 5 spinally intact rodents were used to compare the efficacy of frame-based electrode delivery to free-hand delivery into the spinal cord. Each rat was housed individually under standard conditions on 12-hour light/dark cycles with ad libitum access to water and food. All procedures were conducted in accordance with National Institutes of Health guidelines and approved by the Mayo Clinic Institutional Animal Care and Use Committee. A rodent SCI model was selected for this proof-of-concept study, because it retains the functional properties required for limb control and represents an inexpensive alternative to feline and larger animal models.

\section{Rodent Model of SCI}

Eleven animals were used to develop a model of chronic SCI. Each of these animals was anesthetized with ketamine $(80 \mathrm{mg} / \mathrm{kg})$ and xylazine $(5 \mathrm{mg} / \mathrm{kg})$ and placed on a heating pad to maintain core temperature. The general surgical procedure is as follows: The location of T-4 was identified by counting down from the spinous process at $\mathrm{T}-2$. A bilateral laminectomy was performed at T-4 to expose the spinal cord (Fig. 1A). After laminectomy, a complete transection of the spinal cord was performed at the T-4 level. After verification of complete transection, the incision was surgically closed by intramuscular and skin sutures, and the animals rested for 7 days to allow them sufficient time for recovery from spinal shock. During this time, intraperitoneal injections of buprenorphine $(5 \mu \mathrm{g} / \mathrm{kg}$, twice per day) and intramuscular Baytril $(5 \mathrm{mg} / \mathrm{kg}$, twice per day) were administered. In addition, the rodents' urinary bladders were expressed 3 times per day after surgery until reflex voiding was achieved. Passive flexion and extension of the hindlimbs were performed twice daily to maintain joint flexibility and reduce spasticity. A nociceptive paw-pinch test was used to verify complete SCI at the end of the 7-day recovery period.

\section{Intraspinal Microstimulation}

After a recovery period of 7 days for 9 animals with SCI or on Day 1 for the spinally intact animals, the lumbar spinal cord of each rat was exposed via bilateral laminectomy and durotomy from L-1 to L-4 (Fig. 1A). The spine and hip were stabilized with a spinal clamp and hip pins while allowing free movement of the hindlimbs. Tefloninsulated $90 \%$ platinum-10\% iridium stimulating microelectrodes (each $50 \mu \mathrm{m}$ in diameter with a 30 - to $60-\mu \mathrm{m}$ exposed tip), targeting the ventral horn gray matter, were inserted manually into the spinal cord (Fig. 1B) at an approximate depth of $1.8 \mathrm{~mm}$ from the dorsal surface of the cord. ${ }^{30}$ The initial electrode insertion was performed approximately $1 \mathrm{~mm}$ lateral to the midline, starting at the caudal level of the L-2 vertebra. If a desired hindlimb response was not achieved, the electrode was removed and reinserted caudally in 500- $\mu \mathrm{m}$ increments to the rostral region of L-4. This region of the spinal cord was targeted for evoking hindlimb movements, according to previous literature. ${ }^{1,30}$ A return electrode was inserted into the lateral abdominal muscle. Stimulation parameters were controlled using a wireless neurostimulation system (described below). The stimulation amplitude was increased linearly at each stimulating electrode location (from 10 $\mu \mathrm{A}$ to $100 \mu \mathrm{A}$ ) while the pulse width and frequency were 


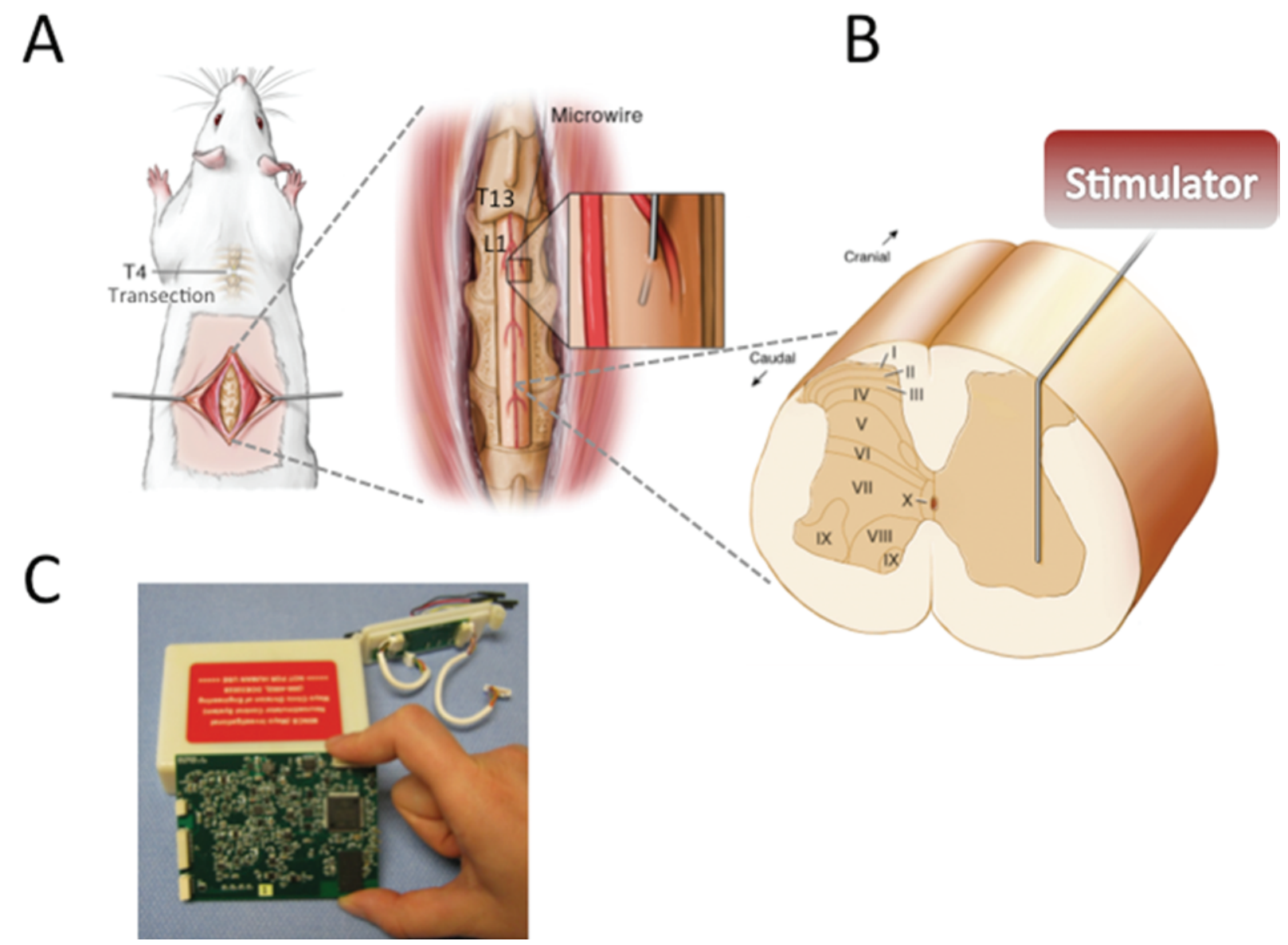

FIG. 1. A: Schematic representation of the rodent ISMS model. The spinal cord was transected at the T-4 level. A bilateral laminectomy was performed from L-1 to L-4 to expose the dorsal surface of the spinal cord. B: Schematic representation of a typical microelectrode implantation into the ventral gray matter of the right lumbar hemicord. C: The MINCS for wireless control of the stimulation. Copyright J. Luis Lujan. Published with permission.

sustained at $0.2 \mathrm{msec}$ and $25 \mathrm{~Hz}$, respectively, until predefined target hindlimb movements (i.e., hip extension, hip flexion, knee extension, knee flexion, ankle extension, and ankle flexion) were evoked by stimulation. When a target movement was achieved, the microelectrode was fixed to the spinal cord using cyanoacrylate adhesive. Otherwise, electrode insertion and microstimulation were repeated at the next spinal location. All stimulation studies were acute and lasted no more than 8 hours, in accordance with Institutional Animal Care and Use Committee protocol.

\section{Wireless Neurostimulation System}

A proprietary neurostimulation system, the Mayo investigational neuromodulation control system (MINCS), ${ }^{5}$ was used to provide wirelessly controlled ISMS (Fig. 1C). The MINCS was originally designed to provide deep-brain stimulation synchronized with a wireless instantaneous neurotransmitter concentration sensor (WINCS) to eliminate stimulus artifacts. ${ }^{5,9,18}$ Front-end analog circuitry with voltage-regulated and current-regulated drivers generate voltage- and current-controlled stimuli on up to 4 channels. A 32-bit microcontroller (Stellaris LM3S9B96; Texas Instruments) and a Bluetooth transceiver (LMX9838; Texas Instruments) provide wireless control of the stimulation. A 10-bit analog-to-digital converter is used to measure the amplitude of the applied stimulus and to periodically verify battery capacity. To produce a stimulation sequence, the microcontroller adjusts a 14-bit digital-to-analog converter and directs its output signal to either the voltage-regulated or current-regulated driver, each of which is based on the LM8261 operational amplifier (National Semiconductor). An array of analog switches steers the stimuli emitted by the amplifier to the selected electrodes. The output stimulation range is $50 \mathrm{mV}$ to $10 \mathrm{~V}$ in voltage-regulated mode and $10 \mu \mathrm{A}$ to $10 \mathrm{~mA}$ in current-regulated mode, which provides adequate compliance for a wide range of electrode impedances. Pulse duration can vary between 0.05 and 2 msec. Pulse patterns can be either monophasic or biphasic, and the applied charge can be balanced asymmetrically. To improve accuracy, an automated calibration procedure compensates for offsets, variances, and internal impedances in the analog signal path. A second calibration procedure improves the accuracy of the analog-to-digital converter by measuring the stimulus voltage and current. Developed as a prototype for a next-generation stimulator for use in humans, analog circuitry in the MINCS allows voltage and impedance sensing to detect electric faults and to implement protection mechanisms intended to ensure patient safety. The system is built on a multilayer printed 
circuit board and powered by a rechargeable 6.5 -watt-hour lithium-ion battery (Ultralife). The wide operating range of the MINCS enables its use for neurostimulation of the spinal cord, brain, and muscle tissue. The control software, written in Microsoft Visual Studio C\#, allows the user to modify the stimulation parameters remotely. A wide range of custom-designed stimulus sequences can be used to deliver monophasic and biphasic stimuli. The software can direct stimulation to any combination of the 4 electrode channels. In addition, the stimulator and software system can calculate electrode impedance in vivo and provide real-time information about the integrity of each electrode by measuring the applied voltage and current flow through the tissue load.

\section{Intraspinal Stimulation Paradigms}

To establish a wireless platform for developing and testing novel spinal stimulation paradigms for restoring motor function, we examined the feasibility of wirelessly controlling hindlimb muscles in a rodent SCI model and in healthy anesthetized controls. The stimulation paradigms selected (Table 1) were designed to evoke basic motor responses representing the building blocks required for complex hindlimb function. These paradigms were defined as selective control of muscle activation via graded control of flexion/extension responses, fatigue-resistant activation via sustained hip extension, and repeatable bilateral responses. Each stimulation paradigm was repeated 5 or 10 times in each subject $(5$ times for bilateral responses, 5 times for sustained activation, and 10 times for graded responses). Hip, knee, and ankle joint angle baselines were determined before and after stimulation for each paradigm. For the case of graded flexion/extension response, we used a ramped-stimulation paradigm. In this paradigm, we stimulated at L-3 using 0.2-msec pulses at $25 \mathrm{~Hz}$. Amplitude was increased from 0 to $100 \mu \mathrm{A}$ and decreased back to 0 by using a $10-\mu \mathrm{A}$ step size. Stimulation at each amplitude was sustained for 0.8 seconds followed by 30 seconds of rest. In the case of sustained activation, we stimulated at L-3 using a custom trapezoidal paradigm with a $100-\mu \mathrm{A}$ plateau amplitude and a $4-\mu \mathrm{A}$ step size for the ramp-up and ramp-down phases. Stimulation pulses in the ramp-up and ramp-down phases of this paradigm were $45 \mathrm{msec}$ in duration, followed by a 10-msec rest interval. The plateau phase was sustained for $220 \mathrm{msec}$ by using the pulse duration and rest interval described for the ramp edges. For the repeatable response paradigm, we stimulated bilaterally at L-3 to extend and relax both hindlimbs in a cyclic alternating sequence. Only 1 animal with SCI and 1 healthy control were included in this paradigm. Stimulation was performed at $25 \mathrm{~Hz}$ using a $0.2-\mathrm{msec}$ pulse duration and a 50- $\mu \mathrm{A}$ pulse amplitude.

TABLE 1. Stimulation paradigms

\begin{tabular}{lccc}
\hline \multirow{2}{*}{ Parameter } & Graded & Sustained & Bilateral \\
\cline { 2 - 4 } Frequency $(\mathrm{Hz})$ & 25 & 18 & 25 \\
\hline Pulse width $(\mathrm{msec})$ & 0.2 & 45 & 0.2 \\
\hline Amplitude $(\mu \mathrm{A})$ & $10-100$ & $0-100$ & 50 \\
\hline
\end{tabular}

\section{Frame-Based Electrode Implantation}

We tested a frame-based method of inserting and securing the microwire electrodes in a cohort of 5 anesthetized animals with neurologically intact spinal cords. Each electrode was inserted into the lumbar region of the spinal cord using a spine frame system (Kopf Instruments) in an attempt to reduce implantation error and maintain electrode position. In each of these rats, individual penetrations into the cord were recorded with respect to spinal location and the movement evoked. Implantation was repeated until a hip extension response was achieved. The electrode was then secured within the spinal cord using the frame system combined with cyanoacrylate adhesive. Once the electrode was secured, the stimulation paradigm followed the same protocol as that of the graded stimulation paradigm listed in Table $1(0-100 \mu \mathrm{A}, 200-\mu \mathrm{sec}$ pulse duration, $25 \mathrm{~Hz}$ ).

\section{Kinematic Analysis}

Opaque markers were placed on the iliac crest, the fifth metatarsal, and the hip, knee, and ankle joints to monitor limb kinematics by using motion analysis. Limb movements of each rat were recorded from a parasagittal perspective at 50 frames/second using two $2048 \times 1088$-pixel ace GigE cameras (Basler) while the animal was mounted on a spinal unit (Kopf Instruments). Kinematic responses were analyzed offline using a Templo 2D motion analysis system (Contemplas). Wireless monitoring of electromyographic (EMG) signals (Trigno wireless EMG; Delsys Inc.) was performed to identify the specific muscle groups and individual muscles activated in response to stimulation. Intramuscular EMG needles were placed in the hamstrings, biceps femoris, and vastus lateralis muscles to evaluate hindlimb movement. Intramuscular EMG data (common mode rejection ratio $>80 \mathrm{~dB}$; gain of 300 ) were recorded at $4000 \mathrm{~Hz}$ across a bandwidth of $20-450 \mathrm{~Hz}$ using a 16-bit Trigno wireless system. The reference electrode was inserted into deep back musculature. EMG data were recorded and transmitted wirelessly to a Windowsbased computer via Bluetooth technology for offline analysis. Kinematic data were analyzed by using MatLab (MathWorks) with a custom-built Windows 7 computer system (zFlo, Inc.) with 2 quad-core i7 processors and 16 GB of RAM. Changes in joint angle were calculated by the subtraction of joint position offsets before stimulation onset from the entire signal. To enable comparisons across different muscles and subjects, the EMG signal amplitude was determined by calculating the root mean square of the sampled signal and normalizing it to the maximum EMG amplitude recorded.

\section{Statistical Analysis}

Least-squares regression analyses of the dependencies of angle changes on stimulation amplitude were performed for the knee, hip, and ankle joints using MatLab. Pulse width and stimulation frequency were not included in the analysis. High stimulation frequencies can achieve smooth contractions at the cost of further muscle fatigue. Therefore, only the minimum frequency that resulted in smooth tetanic contractions was used to minimize muscle 
fatigue. In addition, the net effect of systematically varying the pulse duration is similar to that of performing corresponding changes in stimulus amplitude when all other parameters are kept constant. ${ }^{8}$ Statistical analyses of these dependencies were performed using Student t-test in MatLab. The significance level was set at a $p$ value of $<0.05$.

\section{Verification of Electrode Location}

After each rat was killed, the lumbar spinal cord was surgically removed and the cord tissue was fixed in $4 \%$ paraformaldehyde for 7 days and then embedded in paraffin. The paraffin-embedded tissue was sliced into $15-\mu \mathrm{m}$ axial sections using a rotary microtome (Leica Biosystems). Each histological section was stained with Masson trichrome stain (Sigma-Aldrich Co.). The trajectory of each electrode and its tip location were confirmed using light microscopy.

\section{Results \\ SCI Model}

Complete paralysis below the injury level was confirmed after 7 days in 9 animals that underwent spinal cord transection, as ascertained by the absence of both volitional movements and withdrawal reflexes in response to nociceptive stimuli applied to the hindlimbs.

\section{Wireless Control of ISMS}

The results of the 3 stimulation paradigms (Table 1) tested in this feasibility study demonstrate the ability to use ISMS to evoke and control limb responses remotely at a distance of $10 \mathrm{~m}$. Wirelessly controlled ISMS was successful in remotely evoking repeatable $\left(\mathrm{R}^{2}>0.89, \mathrm{p}\right.$ $<0.001)$ movements at the hip, knee, and ankle joints in $8(90 \%)$ of 9 animals with SCI and $3(100 \%)$ of 3 healthy anesthetized controls (Fig. 2). One animal with SCI failed to respond to intraspinal stimulation and was immediately killed. Wirelessly controlled ISMS evoked graded and sustained tetanic muscle responses involving the hip, knee, or ankle joints, such as those required for standing, in 3 healthy controls (Fig. 3A) and in 1 animal with SCI (Fig. 3B). The activation thresholds required to evoke specific responses in intact controls were similar to those observed in paretic animals $\left(\mathrm{R}^{2}=0.98, \mathrm{p}<0.001\right)$ when stimulation was applied at the same spinal level (i.e., L-3). Furthermore, wirelessly controlled ISMS successfully coordinated bilateral muscle contractions and evoked sustained and repeatable hindlimb movements similar to those observed during locomotion in 1 animal with SCI and in 1 intact animal when each was implanted at the L-3 vertebral level (Fig. 4). Pearson's correlation showed that the stimulation-evoked bilateral responses we observed were repeatable $\left(\mathrm{R}^{2}=0.77, \mathrm{p}<0.001\right)$.

\section{Graded Response to ISMS}

Proportional stimulation-evoked hindlimb extension and flexion movements were observed for a wide range of stimulus amplitudes (Table 1) in $90 \%$ of the animals with SCI and in $100 \%$ of the healthy controls (Fig. 2). The stimulation-evoked responses to this stimulation para- digm were quantified by measuring angle changes (from baseline) at the hip, knee, and ankle joints. Change in joint angle as a function of stimulus intensity was similar for intact animals and those with SCI $\left(\mathrm{R}^{2}>0.8, \mathrm{p}<0.001\right)$. During flexion responses, peaks in angle change were observed at $70 \mu \mathrm{A}$ for the ankle joint and $80 \mu \mathrm{A}$ for the knee joint, with stimulation beyond $80 \mu \mathrm{A}$ resulting in a decrease in angle (Fig. 2A and B). During hindlimb flexion, the hip joint exhibited the lowest change in angle with respect to baseline. In addition, the hip joint required higher stimulation intensities (at least $40 \mu \mathrm{A}$ ) than did the ankle and knee joints ( 20 and $30 \mu \mathrm{A}$, respectively) before joint movement was detected. The magnitudes of the angle changes were correlated directly to the stimulation amplitudes used in this paradigm, with Pearson's correlation coefficients $\left(\mathrm{R}^{2}\right)$ of $0.9,0.8$, and 0.8 for the hip, knee, and ankle joints, respectively ( $<<0.05$ for all 3 joints).

\section{Sustained Response to ISMS}

Increasing stimulation at the L-3 vertebral level resulted in smooth angle changes at the hip joint over 5 trials in 3 intact controls (Fig. 3A) and in 1 animal with SCI (Fig. 3B). In addition, sustained stimulation at $100 \mu \mathrm{A}$ resulted in sustained hip extension (an approximately $40^{\circ}$ change from baseline for the intact animal and a $28^{\circ}$ change for the animal with SCI) over a 2-second stimulation interval. This constant response was followed by a smooth angle change back down to resting state. Maximum hip extension was observed at $70 \mu \mathrm{A}$ and remained constant as the stimulation amplitude increased from 70 to $100 \mu \mathrm{A}$. Changes in hip angle during sustained stimulation were directly correlated to the stimulation amplitudes for both paralyzed and control animals $\left(\mathrm{R}^{2}>0.9, \mathrm{p}<0.05\right)$.

\section{Bilateral Response to ISMS}

Alternating stimulation to the left and right hemicords at the L-3 level evoked controlled, sustained, and repeatable bilateral hip extension in 1 rat with SCI and in 1 intact control. The stimulation sequence applied to the right hemicord was delayed by 2.5 seconds with respect to the sequence applied to the left hemicord to generate an alternating response (Fig. 4). The changes in hip angle during bilateral alternating stimulation were larger for the left hip joint than for the right in the animal with SCI, suggesting asymmetries in the electrode locations. In addition, the angle change measured at the right hip decreased over 5 stimulation cycles, suggesting possible fatigue onset. This response was not observed at the left hip joint. Both left and right hip angle changes were directly correlated to stimulation amplitude, with $\mathrm{R}^{2}$ correlation coefficients of 0.98 and 0.99 for the left and right hip joints, respectively $(\mathrm{p}<0.001)$. Alternating stimulation was successfully applied to 1 intact animal. However, a failure with the motion-caption system prevented quantification of the evoked motor responses.

\section{Graded Response After Frame-Based Electrode Delivery}

Frame-based electrode implantation via dorsal landmark targeting was equally effective at evoking hip extension as free-hand electrode delivery (Fig. 5). Hindlimb movements resulting in hip extension were initially observed when a stimulus of $40 \mu \mathrm{A}$ was applied to the L-3 

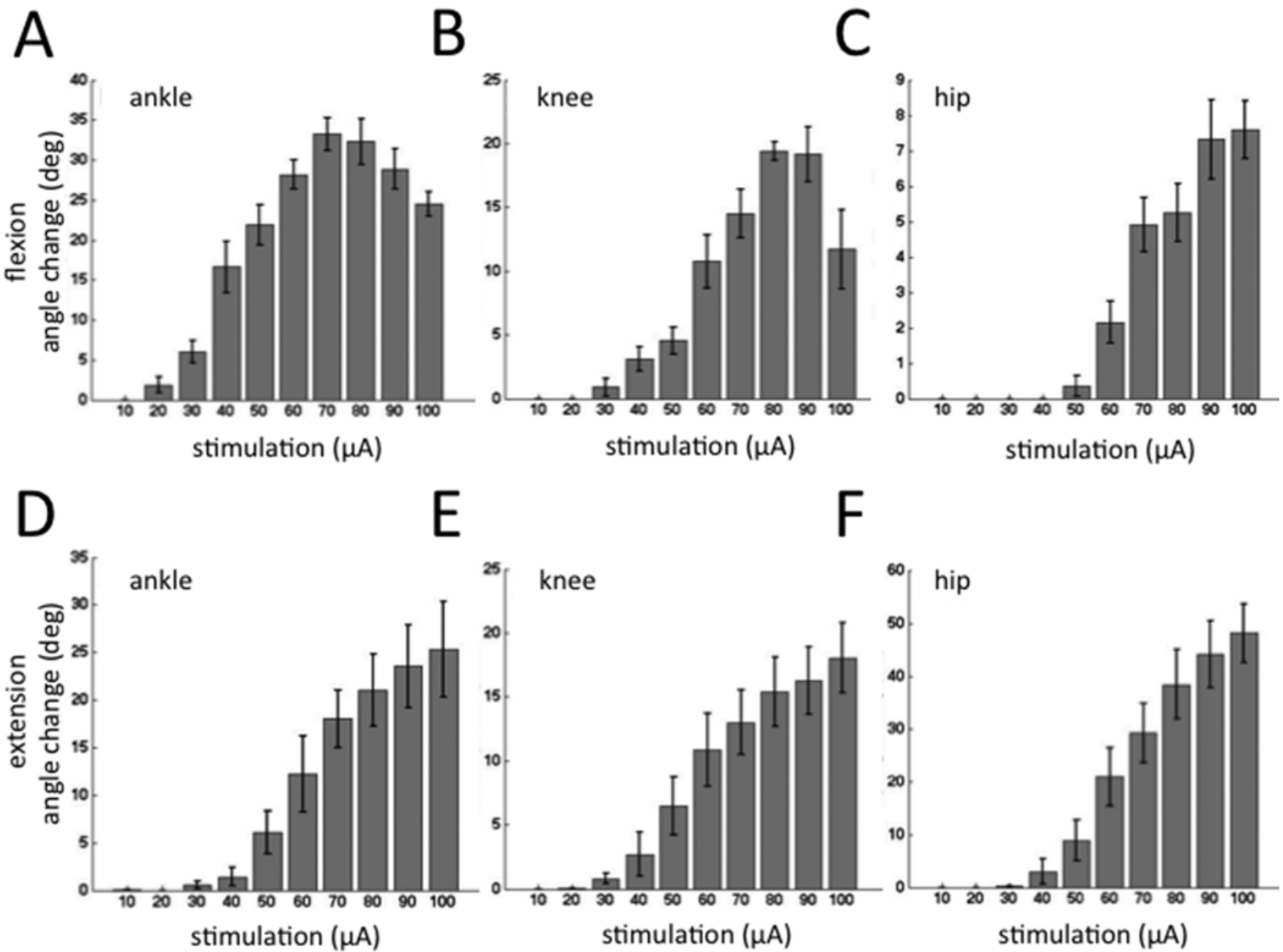

FIG. 2. Shown are the graded changes in joint angle as a function of stimulation amplitude for flexion of the ankle (A), knee (B), and hip joints (C) and as a function of stimulation amplitude for extension movement of the ankle (D), knee (E), and hip joints (F). Pulse width and frequency were held constant at $0.2 \mathrm{msec}$ and $25 \mathrm{~Hz}$, respectively. Data shown represent the mean and SD from 8 animals with $\mathrm{SCl}$ and 3 controls. deg $=$ degrees.

region of the spinal cord, and tetanic responses for each technique were observed at $100 \mu \mathrm{A}$. A statistical comparison of each technique showed significant differences between the mean joint angle changes at each stimulus intensity ( $\mathrm{p}<0.0031$, Student t-test). In addition, histological analyses of spinal cord specimens $(\mathrm{n}=3$ for free-hand electrode implantation and $\mathrm{n}=3$ for frame-based electrode delivery) showed higher numbers of electrode placements within the ventral horn region of the lumbar spinal cord for free-hand electrode delivery (12 histological confirmations) than for frame-based delivery (5 histological confirmations) (Fig. 6). Conversely, free-hand delivery required a larger number of penetrations (21 electrode penetrations) in these 3 animals to evoke the desired hip extension movement (compared with 10 electrode penetrations in the 3 animals in which frame-based delivery was used) (Fig. 6).

\section{Discussion}

It has been suggested that ISMS recruits motor units according to Henneman's size principle, thereby offer- ing better resistance to muscle fatigue and lower activation thresholds than other electrical stimulation techniques. ${ }^{1,22,23,27}$ However, technological limitations have restricted this promising technology to research laboratories. Therefore, it is necessary to develop novel stimulation technology that will facilitate the use of ISMS outside of the restrictions imposed in the laboratory setting. In this proof-of-principle study, we present a wireless ISMS platform for developing and testing novel stimulation paradigms and technologies that will ultimately lead to simultaneous stimulation and behavioral analysis without the restrictions imposed by testing within a laboratory environment.

The results presented herein show that wireless ISMS using the MINCS was successful at producing graded, sustained, and repeatable activation of single and multiple synergistic muscles, which are required for generating complex movements such as standing and walking. The stimulator device described herein represents an alternative for providing wireless control of stimulation. However, its wide operating range enables us to take advantage of 

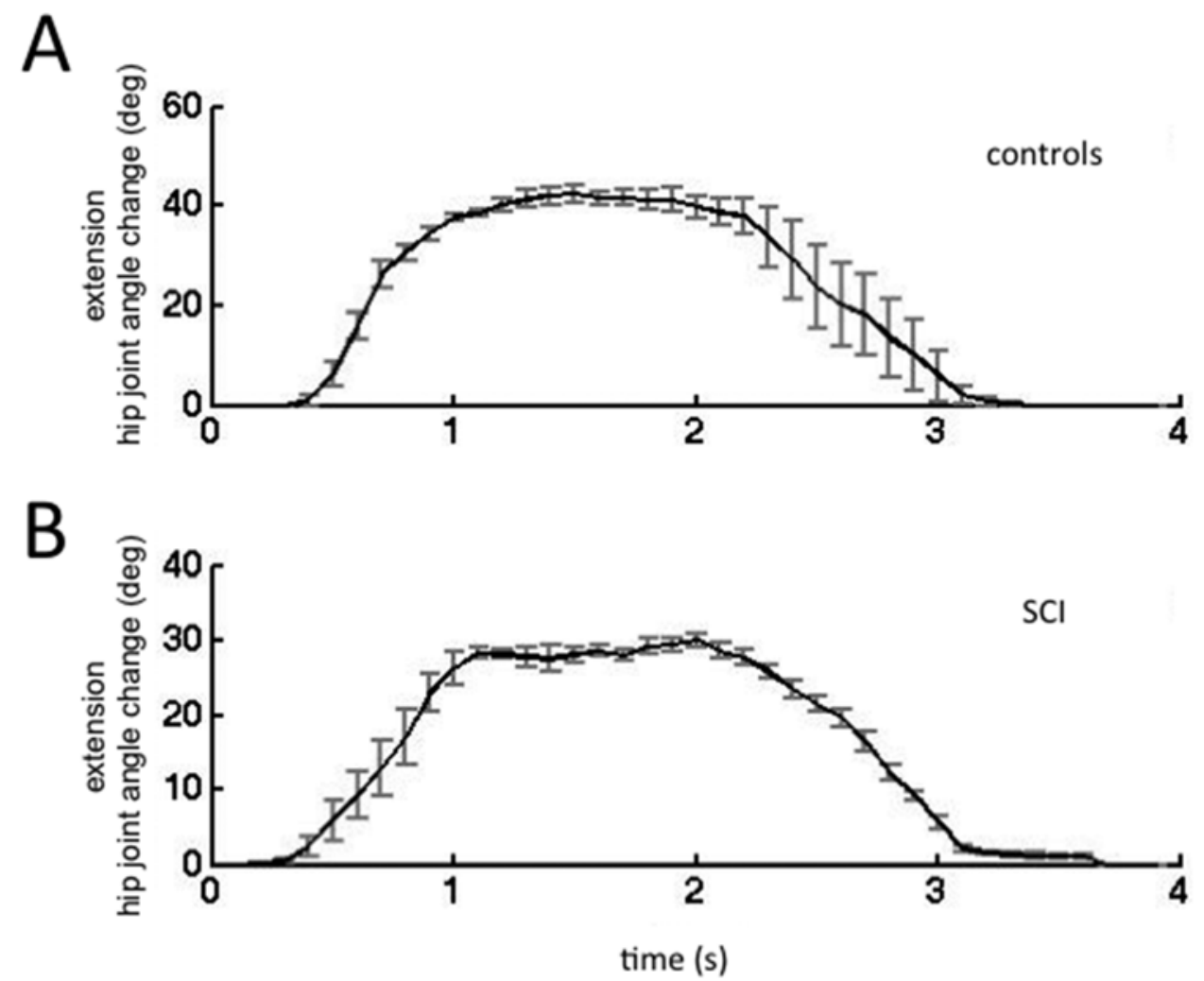

FIG. 3. Hip extension evoked by graded stimulation in 3 intact controls (A) and 1 animal with $\mathrm{SCI}(\mathrm{B})$. Each trial was repeated 5 times. Data shown represent the mean and SD. $(s)=$ seconds.

its wireless capabilities for remote stimulation of spinal cord, brain, and muscle tissue. Wireless control of limb movement using Bluetooth technology was successful at distances of up to $10 \mathrm{~m}$, the maximum distance attempted, without any loss of signal quality or data-transmission errors. It must be noted that Bluetooth radios have been shown to work at distances $>1$ mile away with the aid of directional antennas and signal amplifiers, which creates many opportunities for testing ISMS technology in a wide range of environments. However, careful consideration must be given to the security and safety of patients before wireless technology is used to drive neural stimulators in a clinical setting. The MINCS was designed originally for stimulating the brain (i.e., deep-brain stimulation) in human patients. As such, it was built from the ground up with patient safety in mind. Furthermore, it offers the capability of wireless synchronization with other devices (e.g., neurotransmitter and electrophysiology sensors), which in turn provides the opportunity for developing novel brainmachine interfaces capable of optimally restoring function after SCI.

Graded, sustained, and repeatable hindlimb movements were evoked successfully using wireless ISMS and freehand delivery of electrodes in 3 control animals (100\%) and 8 animals with SCI (90\%). The responses evoked by each stimulation paradigm were similar in the control and SCI populations $\left(\mathrm{R}^{2}>0.89, \mathrm{p}<0.05\right)$. Controlled extensions of the ankle and knee joints were achieved at stimulation amplitudes as low as 20 and $30 \mu \mathrm{A}$, respectively. Stimulation amplitudes of $>80 \mu \mathrm{A}$ resulted in decreased extension angles for the graded hindlimb flexion paradigm (Fig. 2), which can be explained by suprathreshold stimulation and subsequent spillover to adjacent antagonist motor neuron pools. It is interesting to note that spillover was not observed when hindlimb extension responses were targeted (Fig. 2D-F), which can potentially be explained by slight differences in electrode placement within the spinal cord or by the larger area of the L2-4 spinal cord lumbar enlargement region associated with motor neurons controlling hindlimb extensor muscles compared with flexors. Unfortunately, a low signal-to-noise ratio prevented us from obtaining reliable wireless EMG data. Bilateral stimulation at the L-3 level in 1 animal with SCI and in 1 intact animal resulted in the repeatable activation of hip extensors. To examine the feasibility of using the MINCS to control bilateral limb function and establish proof of principle with no crossover effects, only 1 animal from each group was tested under this paradigm. A failure of the motion-analysis system prevented analysis of kinematics in the intact animal. In the animal with SCI, the magnitude of the limb movement for the right hip was lower than that on the left, which can be explained by cord asymmetry and electrode positioning variability in both the left and right hemicords. In addition, repeated hip extension on the right limb shows decreasing amplitude as a function of time, suggesting the possibility of fatigue or electrode migration. Future studies using bilateral stimulation should focus on a 4-electrode configuration to evoke bilateral flexor and extensor movements to establish wireless control of functional stepping. 


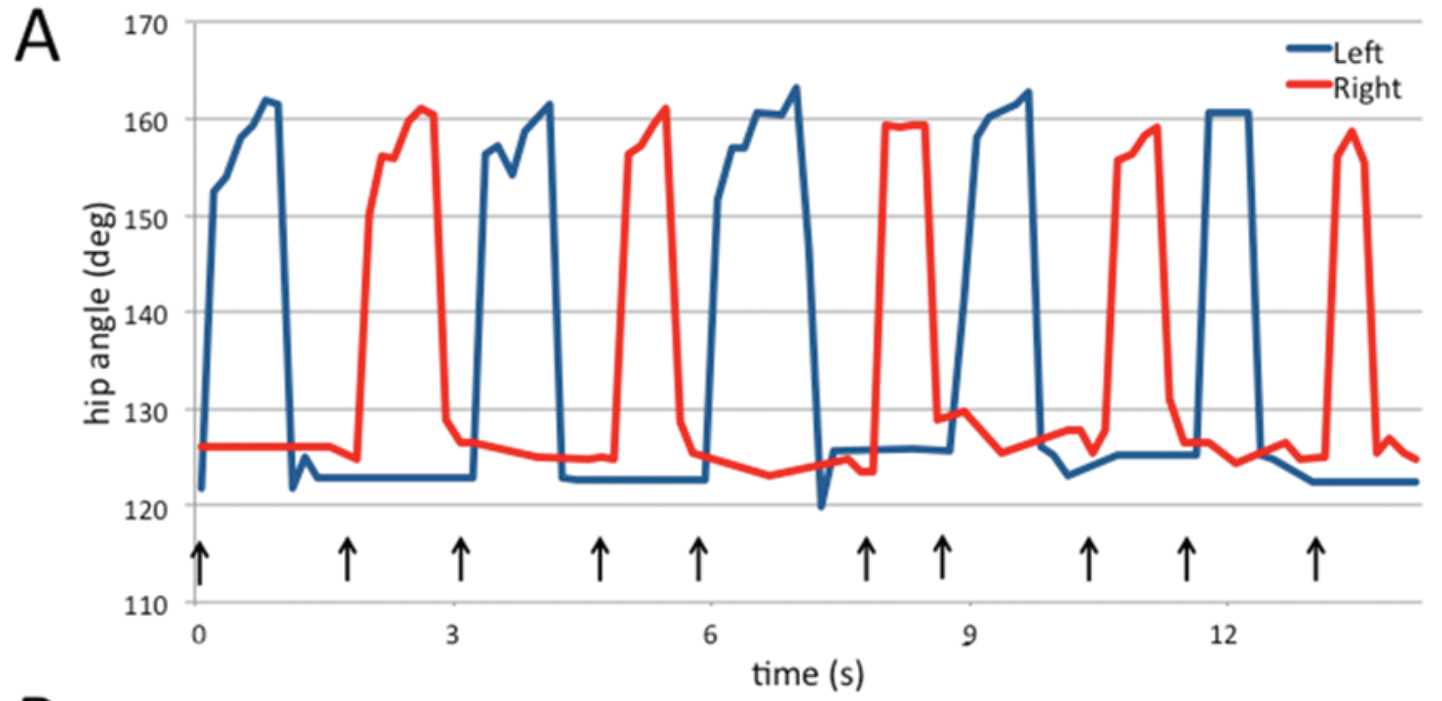

B

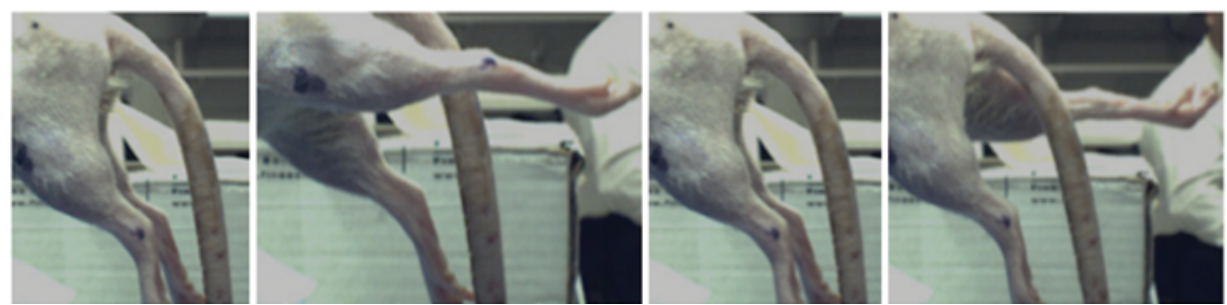

FIG. 4. A: Kinematic analysis of hip responses evoked by alternating bilateral stimulation at the L-3 level. The blue trace represents the hip extension angle evoked in the left hindlimb. The red trace represents the hip extension angle evoked in the right hindlimb. Arrows represent stimulation onset. Pulse width and frequency were held constant at $0.2 \mathrm{msec}$ and $25 \mathrm{~Hz}$, respectively. B: Limb movements achieved with alternating stimulation of motor neurons at L-3. From left to right: resting state, maximal hip extension of the left hindlimb, resting state, and maximal hip extension of the right hindlimb. Figure is available in color online only.

Implantation of electrodes outside the ventral motor neuron pools still successfully evoked the desired hip movements with no statistically significant difference in stimulation-evoked responses between groups (Fig. 5). In addition, the use of a frame-based system reduced the number of electrode penetrations required to evoke a desired movement. Altogether, we can conclude that framebased electrode delivery increased the efficiency of evoking a desired movement via stable electrode delivery and fixation; however, efficiently targeting the ventral horn motor neuron region of the spinal cord was not possible using dorsal spinal cord landmarks.

It must be noted that this proof-of-principle study comes with several limitations. First, the stimulation paradigms evaluated in this study did not result in ambulation in awake animals. Instead, the motor responses evoked by each paradigm represent the building blocks for complex hindlimb function. These paradigms were chosen because each possesses basic attributes required for complex functional locomotion. Specifically, graded flexion/extension responses provide selective control of muscles required for achieving major limb movements such as those involved in locomotion (e.g., simultaneous ankle and knee flexion followed by hip flexion required for leg swing). Similarly, sustained extension responses demonstrate the ability to evoke smooth, controlled, and fatigue-resistant responses, such as those required for standing and long-term locomotion. In addition, the bilateral stimulation paradigm established the ability to simultaneously control bilateral movements required for locomotion. Furthermore, our targeting was not intended to activate central pattern generators. As such, providing locomotion with 4 channels of stimulation was not feasible. Moreover, the animal population combined spinalized and neurologically intact animals, which prevented us from performing the experiments under awake conditions.

Second, our study focused on a small-animal model of SCI. However, it is imperative to recognize that small-animal models of ISMS are inadequate for successful translation of functional restoration therapies in humans. ${ }^{19,20}$ Furthermore, these models complicate surgical targeting of the motor pools responsible for evoking specific muscle contractions. Specifically, difficulties with securing the electrode in place and securing the small animal during stimulation-evoked hindlimb movements led to small electrode movements with respect to the targeted neuronal population, even after successful implantation into the target location. These movements occurred approximately once in each animal. Once electrode movement occurred, an average of 2 additional penetrations were required to evoke the original target movement. Electrode movement occurred randomly, with instances at initial stimulation, 


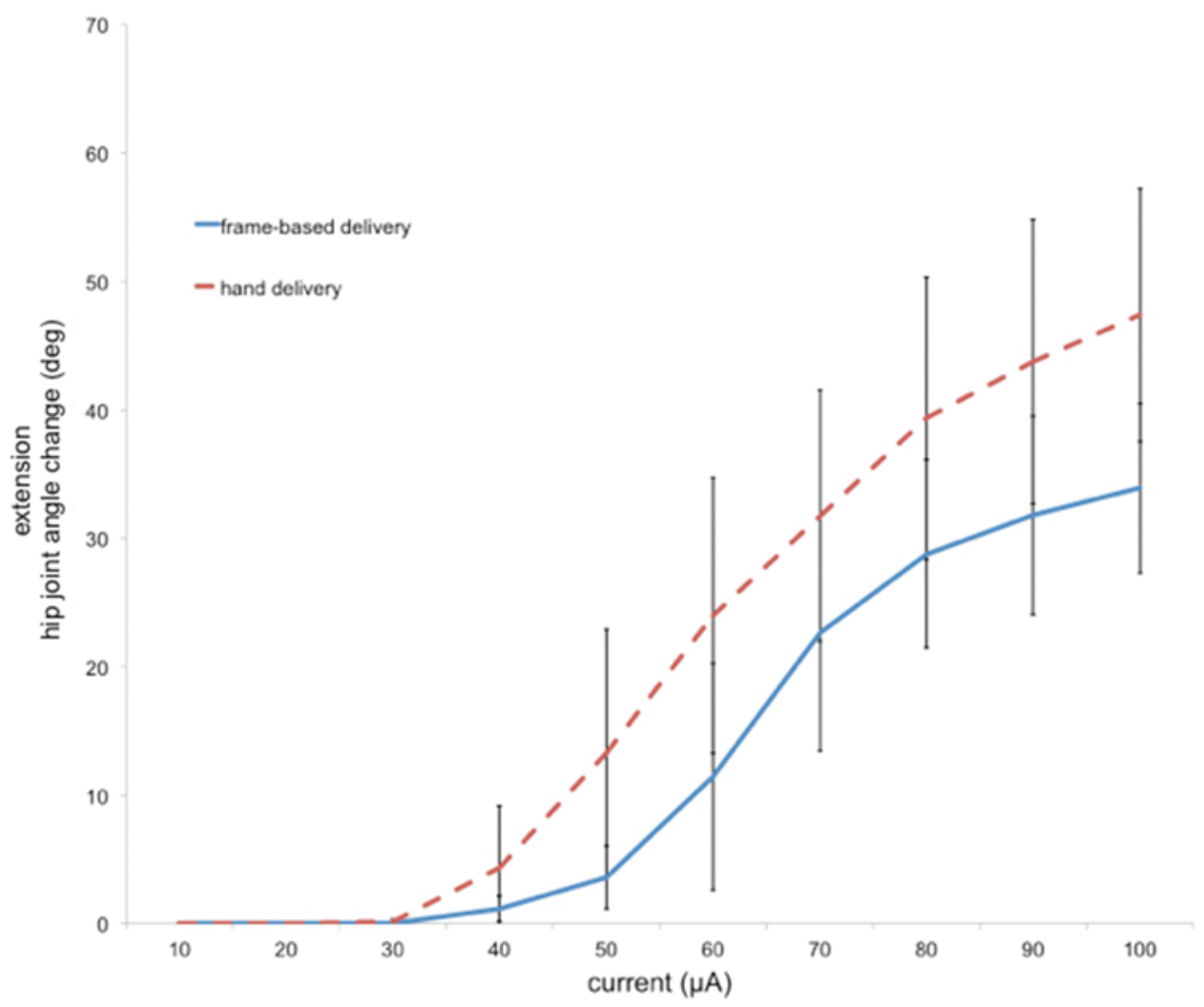

FIG. 5. A comparison of evoked hip extension responses when frame-based (blue line) or free-hand (red line) electrode delivery was used. Mean hip joint angles are shown with respect to stimulation intensities. Error bars indicate the SDs for the free-hand (n $=4$ ) and frame-based $(n=5)$ delivery experiments. Figure is available in color online only.

while securing the electrode, and after securement. We minimized electrode movement by reducing stimulation amplitudes to the minimum amount necessary to evoke the desired movements. However, this process restricted the number of stimulation parameters we were able to apply during stimulation.

The recent development of a large-animal model of spinal stimulation showed that it is possible to target neuronal pools responsible for controlling a specific hindlimb function at more than 1 location along the vertebral column (e.g., rostral L-3 or caudal L-3). This model more closely resembles the human spinal cord anatomy and physiology and will improve targeting and mapping of specific motor pools in the spinal cord for optimizing electrode position. ${ }^{11}$ As such, future efforts should use a model that best represents the neuroanatomical and physiological variance found in the human spinal cord.

Third, in this study, we relied on anatomical landmarks on the dorsal aspect of the spinal cord to guide electrode implantation. Although this practice has been proven successful for evoking movements, histological analysis showed significant deviation of the electrode trajectory from its intended path during electrode insertion. These trajectory deviations and targeting errors can result in the activation of antagonist or other undesired motor neuron populations. ${ }^{28}$ Furthermore, not every stimulating electrode location evoked a movement response. As such, multiple electrode penetrations were sometimes required to achieve a target movement. Each electrode penetration ex- tends surgery time and increases the risk of further damage to the spinal cord. ${ }^{2}$ Therefore, the use of image-guided stereotactic electrode implantation might be necessary to ensure accurate and safe targeting and electrode delivery into the target regions.

Fourth, this study relied on rigid microelectrodes. These electrodes offer improved targeting but can damage the cord if implanted chronically. In this study, electrode migration was mitigated by using cyanoacrylate glue. Future work should focus on developing flexible biocompatible electrodes that minimize tissue reactions and tissue damage and, thus, are suitable for chronic stimulation.

\section{Conclusions}

Successful application of spinal stimulation therapy in humans will require the use of versatile chronic neurostimulation approaches such as wireless systems for behavioral evaluation of novel neurostimulation paradigms. Combining wireless stimulation with image-guided stereotactic delivery systems and flexible microelectrode arrays will enable the selective muscle activation required for functional control of complex multijoint movements. As this technology moves forward, wireless stimulation will provide the ability for paraplegic patients to ambulate freely, with no time or distance limitations associated with current state-of-the-art neurostimulation systems. This technology will also take advantage of telemedicine and allow clinicians to adjust stimulation paradigms over 


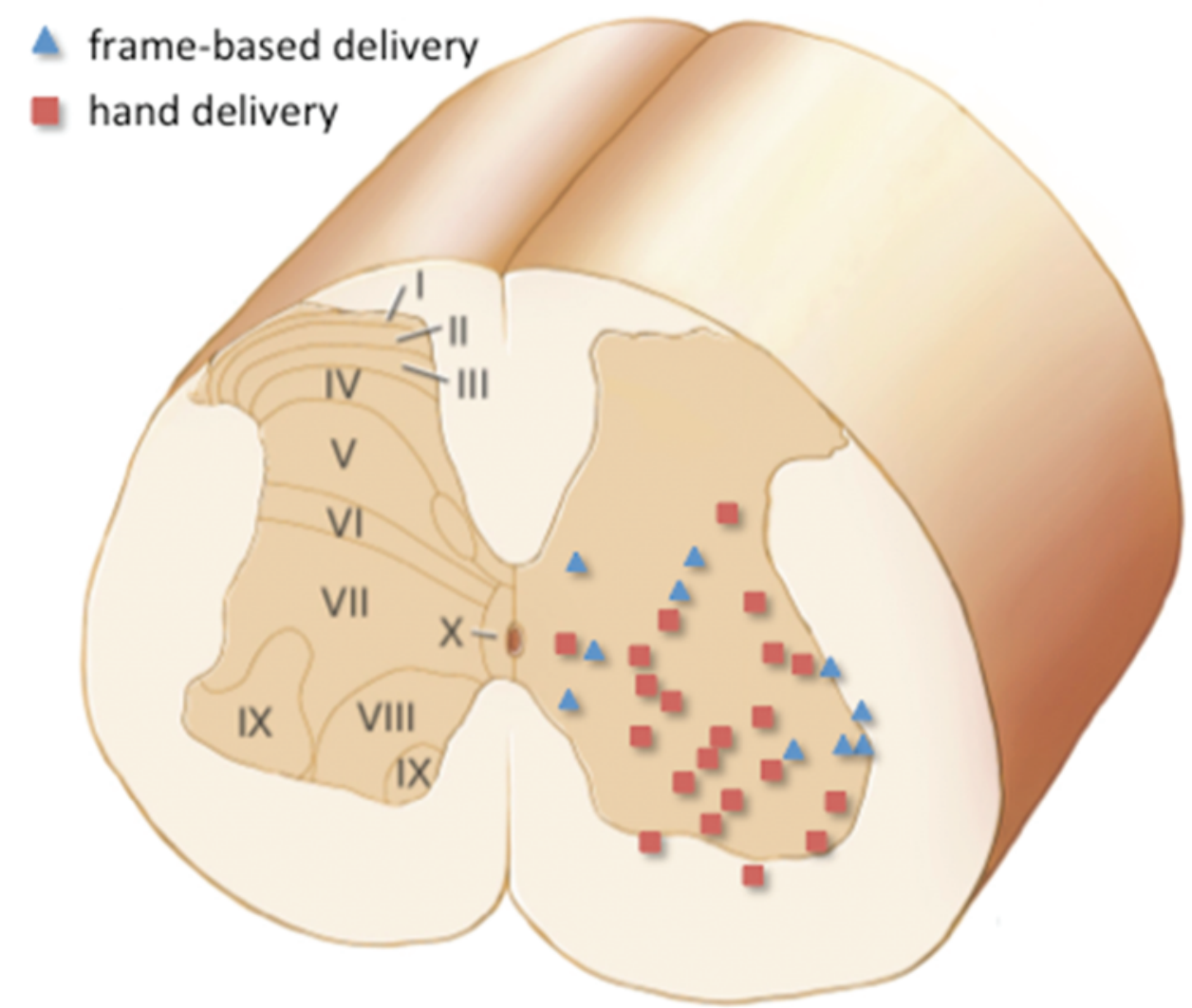

FIG. 6. Electrode location (only the tip location is shown) identified during histological analysis for the free-hand (red squares; $n=$ 3 animals) and frame-based (blue triangles; $n=3$ animals) delivery techniques. Multiple electrode implantations were required in each animal to evoke the target motor responses. Copyright J. Luis Lujan. Published with permission. Figure is available in color online only.

wireless networks when patients are hundreds or even thousands of miles away. Ultimately, the wireless ISMS approach presented herein describes the first steps toward enhancing independence and quality of life for individuals with chronic paralysis.

\section{Acknowledgments}

We thank Mr. Joshua Boesche for his assistance with MINCS programming. We also thank Ms. Ann Schmeichel and Ms. Erika Ross for their assistance with histochemical analysis.

\section{References}

1. Bamford JA, Putman CT, Mushahwar VK: Intraspinal microstimulation preferentially recruits fatigue-resistant muscle fibres and generates gradual force in rat. J Physiol 569:873884,2005

2. Bamford JA, Todd KG, Mushahwar VK: The effects of intraspinal microstimulation on spinal cord tissue in the rat. Biomaterials 31:5552-5563, 2010

3. Bransford RJ, Chapman JR, Skelly AC, VanAlstyne EM: What do we currently know about thoracic spinal cord injury recovery and outcomes? A systematic review. J Neurosurg Spine 17 (1 Suppl):52-64, 2012

4. Butler JE, Lim J, Gorman RB, Boswell-Ruys C, Saboisky JP, Lee BB, et al: Posterolateral surface electrical stimulation of abdominal expiratory muscles to enhance cough in spinal cord injury. Neurorehabil Neural Repair 25:158-167, 2011
5. Chang SY, Kimble CJ, Kim I, Paek SB, Kressin KR, Boesche JB, et al: Development of the Mayo Investigational Neuromodulation Control System: toward a closed-loop electrochemical feedback system for deep brain stimulation. J Neurosurg 119:1556-1565, 2013 (Erratum in J Neurosurg 120:1258, 2014)

6. Crago PE, Peckham PH, Thrope GB: Modulation of muscle force by recruitment during intramuscular stimulation. IEEE Trans Biomed Eng 27:679-684, 1980

7. DiMarco AF, Kowalski KE, Geertman RT, Hromyak DR: Lower thoracic spinal cord stimulation to restore cough in patients with spinal cord injury: results of a National Institutes of Health-sponsored clinical trial. Part I: methodology and effectiveness of expiratory muscle activation. Arch Phys Med Rehabil 90:717-725, 2009

8. Graham GM, Thrasher TA, Popovic MR: The effect of random modulation of functional electrical stimulation parameters on muscle fatigue. IEEE Trans Neural Syst Rehabil Eng 14:38-45, 2006

9. Griessenauer CJ, Chang SY, Tye SJ, Kimble CJ, Bennet KE, Garris PA, et al: Wireless Instantaneous Neurotransmitter Concentration System: electrochemical monitoring of serotonin using fast-scan cyclic voltammetry-a proof-of-principle study. J Neurosurg 113:656-665, 2010

10. Gustafson KJ, Creasey GH, Grill WM: A urethral afferent mediated excitatory bladder reflex exists in humans. Neurosci Lett 360:9-12, 2004

11. Hachmann JT, Jeong JH, Grahn PJ, Mallory GW, Evertz LQ, Bieber AJ, et al: Large animal model for development of functional restoration paradigms using epidural and intraspinal stimulation. PLoS ONE 8:e81443, 2013 
12. Harkema S, Gerasimenko Y, Hodes J, Burdick J, Angeli C, Chen Y, et al: Effect of epidural stimulation of the lumbosacral spinal cord on voluntary movement, standing, and assisted stepping after motor complete paraplegia: a case study. Lancet 377:1938-1947, 2011

13. Henneman E: The size-principle: a deterministic output emerges from a set of probabilistic connections. J Exp Biol 115:105-112, 1985

14. Jackson A, Zimmermann JB: Neural interfaces for the brain and spinal cord-restoring motor function. Nat Rev Neurol 8:690-699, 2012

15. Kasten MR, Sunshine MD, Secrist ES, Horner PJ, Moritz CT: Therapeutic intraspinal microstimulation improves forelimb function after cervical contusion injury. J Neural Eng 10:044001, 2013

16. Kennelly MJ, Bennett ME, Grill WM, Grill JH, Boggs JW: Electrical stimulation of the urethra evokes bladder contractions and emptying in spinal cord injury men: case studies. J Spinal Cord Med 34:315-321, 2011

17. Kilgore KL, Hoyen HA, Bryden AM, Hart RL, Keith MW, Peckham PH: An implanted upper-extremity neuroprosthesis using myoelectric control. J Hand Surg Am 33:539-550, 2008

18. Kimble CJ, Johnson DM, Winter BA, Whitlock SV, Kressin KR, Horne AE, et al: Wireless Instantaneous Neurotransmitter Concentration Sensing System (WINCS) for intraoperative neurochemical monitoring. Conf Proc IEEE Eng Med Biol Soc 2009:4856-4859, 2009

19. Lemay MA, Galagan JE, Hogan N, Bizzi E: Modulation and vectorial summation of the spinalized frog's hindlimb endpoint force produced by intraspinal electrical stimulation of the cord. IEEE Trans Neural Syst Rehabil Eng 9:12-23, 2001

20. Lemay MA, Grasse D, Grill WM: Hindlimb endpoint forces predict movement direction evoked by intraspinal microstimulation in cats. IEEE Trans Neural Syst Rehabil Eng 17:379-389, 2009

21. Mushahwar VK, Collins DF, Prochazka A: Spinal cord microstimulation generates functional limb movements in chronically implanted cats. Exp Neurol 163:422-429, 2000

22. Mushahwar VK, Horch KW: Muscle recruitment through electrical stimulation of the lumbo-sacral spinal cord. IEEE Trans Rehabil Eng 8:22-29, 2000

23. Mushahwar VK, Horch KW: Selective activation of muscle groups in the feline hindlimb through electrical microstimulation of the ventral lumbo-sacral spinal cord. IEEE Trans Rehabil Eng 8:11-21, 2000
24. Peckham PH, Knutson JS: Functional electrical stimulation for neuromuscular applications. Annu Rev Biomed Eng 7:327-360, 2005

25. Popović D, Stojanović A, Pjanović A, Radosavljević S, Popović M, Jović S, et al: Clinical evaluation of the bionic glove. Arch Phys Med Rehabil 80:299-304, 1999

26. Sunshine MD, Cho FS, Lockwood DR, Fechko AS, Kasten MR, Moritz CT: Cervical intraspinal microstimulation evokes robust forelimb movements before and after injury. $\mathbf{J}$ Neural Eng 10:036001, 2013

27. Tai C, Booth AM, Robinson CJ, de Groat WC, Roppolo JR: Isometric torque about the knee joint generated by microstimulation of the cat L6 spinal cord. IEEE Trans Rehabil Eng 7:46-55, 1999

28. Tai C, Booth AM, Robinson CJ, de Groat WC, Roppolo JR: Multi-joint movement of the cat hindlimb evoked by microstimulation of the lumbosacral spinal cord. Exp Neurol 183:620-627, 2003

29. Troyk PR, Donaldson NdeN: Implantable FES Stimulation Systems: What is Needed? Neuromodulation 4:196-204, 2001

30. Watson C, Paxinos G, Kayalioglu G: The Spinal Cord: A Christopher and Dana Reeve Foundation Text and Atlas. New York: Academic Press, 2009

31. Wheeler CA, Peckham PH: Wireless wearable controller for upper-limb neuroprosthesis. J Rehabil Res Dev 46:243-256, 2009

\section{Author Contributions}

Conception and design: Lee, Grahn, Kasasbeh, Bieber, Bennet, Lujan. Acquisition of data: Grahn, Kasasbeh, Mallory, Hachmann, Dube, Jeong, Lujan. Analysis and interpretation of data: Lee, Grahn, Kasasbeh, Mallory, Hachmann, Dube, Lobel, Jeong, Lujan. Drafting the article: Lee, Grahn, Mallory, Lobel, Lujan. Critically revising the article: all authors. Reviewed submitted version of manuscript: all authors. Statistical analysis: Mallory, Lujan. Study supervision: Lee, Lujan. MINCS design: Kimble.

\section{Correspondence}

Kendall H. Lee, Department of Neurologic Surgery, Department of Physiology and Biomedical Engineering, Mayo Clinic, 200 First St. SW, Rochester, MN 55905. email: lee.kendall@mayo. edu. 